\title{
LA SIMULACIÓN DENTRO DE LA BIOMIMESIS COMO MEDIO PARA EL DESARROLLO DE APROXIMACIONES A ARTEFACTOS FACILITADORES EN EL DESPLAZAMIENTO HUMANO
}

\author{
SIMULATION INSIDE OF \\ BIOMIMICRY AS A WAY OF \\ DEVELOPING ARTIFACTS THAT \\ FACILITATE HUMAN LOCOMOTION \\ A SIMULAÇÃO DENTRO DA \\ BIOMIMÉTICA COMO MEIO \\ PARA O DESENVOLVIMENTO DE \\ APROXIMAÇÕES A ARTEFATOS \\ FACILITADORES PARA O \\ DESLOCAMENTO HUMANO
}

Por

\author{
Byron Iram Villamil Villar \\ Profesor Asociado \\ Departamento de Diseño \\ Universidad del Valle, Cali, Colombia \\ byron.villamil@correounivalle.edu.co
}

\author{
Miguel Uribe Becerra \\ Profesor Asociado \\ Departamento de Diseño \\ Universidad del Valle, Cali, Colombia \\ angel.uribe@correounivalle.edu.co
}

Resumen: Una aproximación desde las herramientas de la Biomimética permite proponer elementos de análisis y diseño con el fin de reducir el impacto negativo que causa en el medio ambiente la intervención del hombre para su movilidad: intervenir los terrenos en los sistemas actuales de transporte es una condición para la mayoría de mecanismos que basan su funcionamiento en la rueda. Sin embargo, la naturaleza y los sistemas de locomoción animal permiten entrever y validar la posibilidad de desarrollar sistemas alternos que no requieran afectar el medio ambiente ni ajustarse a los parámetros de funcionamiento de la rueda. El presente texto justifica el desarrollo conceptual y práctico de alternativas de locomoción que respondan al principio de desplazamiento mediante patas que crean una huella muy discreta en los terrenos donde operan, y propone un mecanismo aplicable para el desarrollo de artefactos de locomoción mediante patas.

Palabras Clave: Biomimética, locomoción, sostenibilidad, simulación, diseño Industrial. 
Abstract: An approach from the tools of Biomimicry to propose elements of analysis and design in order to reduce the negative impact causing the intervention of man to their mobility in the environment. The intervention of the land for the current transport systems is a condition for the majority of mechanisms which based its operation at the wheel; nature and animal locomotion systems validate the possibility of developing alternative systems that do not require to affect the environment to be conditioned to the operating parameters of the wheel. The text justifies the conceptual and practical development of transportation alternatives that respond to the principle of displacement by legs that create a very unobtrusive footprint on the land where they operate and proposes a mechanism for the development of artifacts of locomotion by legs.

Resumo: Uma aproximação desde as ferramentas da biomimética permite propor elementos de análise e design com o objetivo de reduzir o impacto negativo causado no meio ambiente pela intervenção do homem para sua mobilidade: intervir os terrenos nos sistemas atuais de transporte é uma condição para a maioria de mecanismos baseados na roda. Porém, a natureza e os sistemas de locomoção animal permitem entrever e validar a possibilidade de desenvolver sistemas alternos que não afetem o meio ambiente nem se ajustar aos parâmetros de funcionamento da roda. Este texto justifica o desenvolvimento conceitual e prático de alternativas de locomoção, que respondam ao princípio de deslocação e que baseam-se nas patas animais, as quais criam uma pegada muito discreta nos terrenos onde operam. Além disso, o artigo propõe um mecanismo aplicável para o desenvolvimento de artefatos de locomoção por meio de patas.

Palavras-chave: Biomimética, locomoção, sustentabilidade, simulação design industrial.

\section{La inspiración en el diseño natural}

El Halcón peregrino (Falkoperegrinus) no es más grande que un cuervo y llega a realizar vuelos en picada a mas de 360 kilómetros por hora y vuelos horizontales por encima de los 96 kilómetros por hora; el tiburón Mako (Isurusoxyrinchus) logra velocidades de 124 kilómetros por hora y el Guepardo o Chita (Acinonyxjubatus) puede correr tras su presa a 114 kilómetros por hora al menos por medio kilómetro; la carrera más veloz registrada de un ser humano es de 37 kilómetros por hora en un décimo de kilómetro. ${ }^{1}$ Es decir que el ser humano es 10 veces más lento que el ave más veloz, 3,5 veces más lento que el pez más veloz y 3 veces más lento que el mamífero más veloz y su velocidad máxima no la puede sostener por más de 10 segundos. 


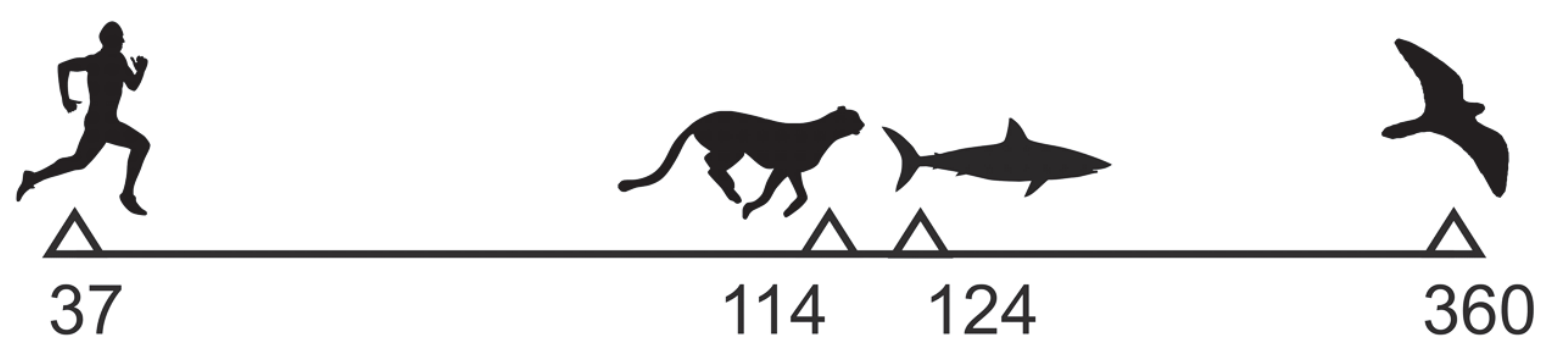

Velocidad máxima en $\mathrm{Km} / \mathrm{hora}$

Figura 1. Comparación de máximas velocidades de algunas especies animales.

No queda otra alternativa que reconocer que el ser humano es muy limitado en términos de desplazamiento y esto sin hacer comparaciones frente a animales que logran mayor altura sobre el nivel del mar, cuáles se sumergen más en las profundidades, cuáles lo pueden hacer por más tiempo o distancia y menos aun con la mayor eficiencia energética en su desplazamiento (Cromer, 1996, pp. 95-120). En pocas palabras el ser humano no está adaptado para destacarse en términos de locomoción, su adaptación al medio ha debido darse bajo parámetros distintos en donde esta capacidad no fuera indispensable, de allí que haya terminado convirtiéndose en sedentario y haya desarrollado la agricultura y la cría de animales.

La debilidad biológica y la pérdida del instinto animal fueron el impulso necesario para el desarrollo de la razón y el pensamiento humano, con lo cual pudo reaccionar, defenderse y adaptarse al medio natural. A los animales, la regulación instintiva les permite adaptarse a su entorno próximo dentro de la realidad percibida, y este modo de adaptación se codifica y es inalterable en el transcurso de su vida, de tal modo que pueden adaptarse a situaciones cambiantes sin entrar a transformar o alterar el entorno y asimilando que son parte de él (Carthy, 1971, p. 17). Es de esta manera como los animales generan un equilibrio armónico entre el código instintivo y el entorno; de otra manera, las cualidades de los seres humanos, como razonar, recordar, crear y mantener pensamientos comprendiendo el presente y previendo un futuro, lo impulsan a percibirse separado del entorno, lo cual entre otras cosas, evidencia sus limitaciones. El ser humano no puede evadir su mente y ella no puede separarse de su cuerpo. En esta dualidad, y forzado a razonar y a reconocer su historia, el ser humano ha registrado un enorme impulso a su razonamiento y, por ende, se ha visto empujado a modificar y adaptar el entorno con el propósito de encajar, pero ya no por medio del instinto, sino pensando y actuando sobre el entorno para su comodidad y estabilidad. 
Gracias a la conciencia de sus limitaciones y a su debilidad, el ser humano desarrolla una capacidad extraordinaria para sobrevivir, el desarrollo del intelecto; la razón, es la esencia del hombre, y junto a ella, como efecto natural de interacción, está la técnica. Maliandi y Thüer (2008) sostienen que el ser humano se define fundamentalmente por su capacidad técnica y explican cómo la conciencia de las debilidades biológicas, que son naturales, las compensa con la técnica, que es expresión de la razón.

El ser humano puede enriquecerse indefinidamente con lo que otros han vivido o por medio de su observación. Esta capacidad se debe a la posibilidad de evocar objetos ausentes y acciones alejadas en la distancia o el tiempo; para ello utiliza el símbolo (como representación de lo real por un signo), cuya forma más elevada es el lenguaje. Así, el ser humano antepone la razón y toda su estructura de comunicación para identificar los signos frente a una eventualidad de la vida, de manera distinta a la reacción instintiva del resto de animales. Como consecuencia de ello, el hombre, cuando afronta un evento de la naturaleza que afecta sus condiciones de vida, en lugar de asumirlo como algo que simplemente sucede y así debe permanecer, busca diferentes formas de transformarlo o, en el mejor de los casos, aprovecharlo para su beneficio.

Como consecuencia del sedentarismo, que tiene antecedentes en las primeras entidades poblacionales por el año 9500 a.C. y la tendencia natural del hombre a transformar y adaptar el entorno, se ha consolidado la ciudad, como espacio adecuado para la permanencia, lo cual conllevó a su vez el desarrollo de la astronomía, las matemáticas, la escritura, el comercio, las sociedades y la cultura. Desde otra perspectiva el ser humano debió adaptar el entorno para su supervivencia y crear un entorno propicio, adecuado a sus capacidades físicas y sus posibilidades sociales, creando espacios para cada actividad como el cobijo, el almacenamiento de alimentos, el procesamiento de los mismos y así, un sin número de espacios específicos y los entornos intermedios que los conectan y organizan. Construyendo un enorme entorno paralelo al entorno natural.

Aun así, con un entorno adecuado para sus necesidades y capacidades el ser humano sigue siendo limitado, y habría que decir también, por el gran desarrollo de estos entornos paralelos, cada vez más grandes, complejos, distantes y diversos, encuentra también en él barreras y dificultades asociadas a sus requerimientos y deseos.

El principal artilugio desarrollado por el hombre para ayudar y hacer más eficiente el desplazamiento en diferentes entornos ha sido la rueda, que se ha usado algo más de cinco mil años según los historiadores, más allá de los aeroplanos y aeronaves de gran complejidad y reciente aparición. Con ella se ha impulsado la movilidad de personas y mercancías con carretas, coches, trenes, 
camiones e infinidad de vehículos diseñados desde su descubrimiento, que de hecho lo han llevado a una nueva forma de nomadismo, muy limitada, pero un poco más allá de sus 4 kilómetros por hora caminando o los 20 kilómetros por hora en una carrera. Sin embargo, la eficiencia de la rueda queda circunscrita al mismo entorno adecuado para el ser humano, salvo pequeñas aventuras off road o pequeños obstáculos en el camino; así se ha hecho necesario inundar sistemáticamente el entorno natural de adecuaciones para que este artilugio pueda rodar y cumplir eficientemente su función, más de 32 millones de kilómetros de carreteras, algo más de un millón cien mil kilómetros de vías férreas ${ }^{2}$ e incontables millones de kilómetros de caminos y vías apropiadas para rodar que dejan una huella imborrable sobre la superficie de la tierra.

Es, por decir poco, preocupante, que en una historia de miles de años el ser humano se haya apegado a un único principio físico de solución para el transporte de personas y carga terrestre, con el que es necesario transformar sistemáticamente el entorno para su operación, dejando de lado los principios sobre los cuales otros seres logran gran eficiencia y velocidad en sus desplazamientos y muy especialmente sin tener que adelantar ninguna adaptación o transformación al medio.

Como estrategia para abordar los problemas de la movilidad, el desplazamiento y el transporte e intentando abandonar las ideas extremadamente complejas como la imitación del vuelo de las aves o la dinámica de los peces en sus respectivos medios ya que son condiciones para las cuales el ser humano se encuentra aun en mayor desventaja por ser eminentemente terrestre, y para encarar a la rueda como transformador del entorno, se ha intentado regresar a revisar los principios del desplazamiento animal por medio de la biomecánica y de la biomimética.

La biomecánica como área de conocimiento interdisciplinaria se ha ocupado especialmente del estudio del ser humano, para ello, "la biomecánica se vale de los conceptos de la física y la ingeniería para describir el movimiento experimentado por los diferentes segmentos corporales y las fuerzas que actúan sobre los mismos durante las actividades normales".

(Nordin \& Frankel, 2001, p. XIII)

Sin embargo Aristóteles (460-370 a.C.) quien podría mencionarse como uno de los primeros en ocuparse del análisis del movimiento, incluyó en sus estudios y en su obra Acerca del movimiento de los animales, la descripción del movimiento y la locomoción, por medio de "un análisis científico de la marcha y un primer análisis geométrico de la acción muscular, así como la fuerza de reacción del suelo" (Millares Marrero \& Puig Cunillera, 1998, p. 4), se ocupó de la locomoción animal y cómo ciertos principios eran comunes y recurrentes como acciones efectivas en el desplazamiento. Más adelante Galeno (131-201 d.C.) diseccionando animales intentaba conocer el funcionamiento del cuerpo humano y escribió De Motu Musculorum sobre los músculos, sus partes y su funcionamiento y así se pudo conocer por medio de descripciones muy precisas las relaciones de causa - efecto en el movimiento. 
Con un gran salto en el tiempo, pasando por la edad media, reaparece el estudio del movimiento con Leonardo da Vinci (1452-1519) definiendo la diferencia entre fuerzas simples y fuerzas complejas, describiendo la anatomía de las articulaciones, representando los movimientos articulares del hombro y la cadera y esquematizando los músculos como líneas de fuerza, y con Vesalio (1514-1564), demostrando los cambios físicos del musculo cuando actúa. Pero fue Giovani Borelli (1608-1679) con su obra De motu animalium quien "integra la fisiología y la física y demuestra mediante métodos geométricos los movimientos humanos como correr, saltar y nadar y la influencia de las fibras musculares sobre el movimiento resultante de contracción" (Millares Marrero \& Puig Cunillera, 1998, p. 5). Sin embargo todos ellos han centrado específicamente su estudio en el ser humano dejando de lado los primeros esfuerzos por hacer este reconocimiento sobre los demás animales. Solo Eadweard Muybridge (1830-1904) financiado por un gran aficionado a la equitación y apoyado en la fotografía estudió el movimiento relativo de los diferentes segmentos articulares; después de esto el desarrollo de la biomecánica se ha centrado casi en exclusiva en el cuerpo humano.

La biónica, ya en el siglo XX, en su definición como ciencia que usa los principios biológicos para el desarrollo de artefactos, ha permitido variadas aproximaciones a las formas de locomoción tomando como referencia a diversos animales y al mismo ser humano. Grandes empresas como Honda y Lockheed Martin han invertido en la investigación y desarrollo de simuladores de la locomoción humana, logrando grandes avances en casi 30 años de trabajo, y esta orientación ha producido logros significativos en la simulación de la locomoción en muchos niveles y podemos ver diversos "Robots" con características particulares, de los que se pueden reseñar los más sobresalientes.

Asimo: (Avanced step in innovative Movility) de Honda. Logra caminar sobre superficies levemente irregulares, subir y bajar escaleras, portar objetos y orientarse en el espacio. ${ }^{3}$

HRP-4: De Kawanda Industries INC en colaboración con el Instituto Nacional de Ciencia Industrial Avanzada y Tecnologías de Japón. Es un humanoide orientado a la interacción en entornos colaborativos con humanos, manipula objetos y se relaciona con el entorno. ${ }^{4}$

NAO: De Aldebaran Robotics, Francia. Humanoide programable y autónomo desarrollado con fines educativos y de investigación, incorpora controladores de código abierto para ser mejorado y transformado. Se usa especialmente en interacciones de enseñanza y apoyo a diversas labores. ${ }^{5}$

Kobian. De Tmsuk y Universidad de Waseda, Japón: Un robot que expresa emociones.

Piper: que percibe gestos y reconoce reacciones de una persona. ${ }^{6}$ 
Atlas, robot Soldado. The Agile Anthropomorphic Robot: Desarrollado por Boston Dynamics para el Departamento de Defensa de los Estados Unidos. Resume todas las capacidades de locomoción y desplazamiento desarrolladas hasta la actualidad. ${ }^{7}$

LS3 Big dog:Legged squad support systems de Boston Dynamics, cuadrúpedo de carga. Es capaz de avanzar en terrenos extremadamente irregulares, desarrollado para la marina de los estados unidos. ${ }^{8}$

T8 De Robugtix: Octópodo impreso en 3D con 26 servo motores potenciado por Big footInverseKinematics. Simula todos los movimientos de locomoción de una araña. ${ }^{9}$

Nano Quadrotors: De Grasplab, Universidad de Pensilvania. Enjambre de robots voladores que simulan enjambres de insectos voladores, que han sido dotados de capacidades de carga y vuelo de alta precisión. ${ }^{10}$

Estos tres últimos retoman los estudios de movimiento y locomoción de los animales, en unos casos artrópodos y cuadrúpedos, en otros de insectos; intentan simular un poco mas allá de los elementos articulares y sus movimientos hasta otras capacidades y cualidades de las especies animales, y se aproximan con ello a la biomímesis.

Es claro que se ha seguido la ruta de la simulación de la bipedestación humana, esperando que los avances cada vez más próximos a su réplica, conduzcan no solo a simular adecuadamente los movimientos humanos sino a mejorarlos. Sin embargo se cree que, si bien sirve como excusa para la investigación y el desarrollo tecnológico, son claras las desventajas de la bipedestación y muy pocas las ventajas que esta forma de locomoción ofrece. Son extremadamente complejos los sistemas de equilibrio y compensación del centro de gravedad para esta forma de desplazamiento y pocas las ventajas comparativas que se obtienen al lograr una pequeña ganancia en altura, aun suponiendo que deben enfrentarse al mismo contexto humano construido. Es claro también que la evolución del cerebro humano acompañó la migración a la bipedestación y esa misma complejidad de desarrollo se hace necesaria en los simuladores.

Encontramos en la naturaleza animales con cerebros muy limitados pero que tienen un desplazamiento exitoso y han sobrevivido miles de años.

El siguiente nuevo paso en la simulación y un nuevo enfoque en la aproximación al estudio de las soluciones naturales se sintetizó en lo que desde los años 90 se denominó Biomimesis; ésta es una nueva mirada frente a los principios naturales y la naturaleza como mentor, es decir, no desde lo que podemos extraer de ella, sino lo que podemos aprender de ella; así mismo, refiere a la naturaleza como medida, como un estándar ecológico para medir las bondades de nuestras innovaciones. Tras 3.8 billones de años de evolución, la naturaleza nos enseña aquello que funciona, aquello que es apropiado y aquello que perdura. 
La biomimética $($ bios $=$ vida; mímesis $=$ imitar) se presenta como una disciplina que adopta el uso práctico de mecanismos, funciones, formas y procesos que estudian las ciencias biológicas, para la aplicación en diversas especialidades, entre ellas el Diseño Industrial.

Uno de sus principales objetivos es fusionar de manera efectiva el concepto de sustentabilidad dentro de la tecnología, con esa clara base biológica y ecológica, promulgando que las leyes y los elementos de la naturaleza son capaces de enseñarnos el camino hacia una evolución en los paradigmas del diseño, asumiendo una perspectiva holística al tratar de emular formas, procesos o estrategias, para conseguir así una verdadera simbiosis al proyectar productos, mensajes o ambientes acordes con los seres vivos, sus interacciones y su biodiversidad.

La biomimética en su breve desarrollo, a pesar de los enormes antecedentes que se pueden reseñar sobre ella, se ha centrado en dos alternativas: de una parte la réplica o la simulación de un organismo integro o partes o componentes particulares de ellos y, de otra parte, el conjunto social y de entorno de una especie, apoyados para los dos casos en la hipótesis de que a través de la evolución los seres vivos alcanzan una perfección funcional que se debe estudiar y hacer útil.

Un sentido más amplio de la biomimética se da cuando su objeto de estudio aborda los ecosistemas integralmente, teniendo en cuenta a los individuos, pero no aisladamente, sino como entes comunitarios que interactúan con otros y con su entorno. Desde este sentido ampliado, Fritjof Capra (1939 -) denomina estar alfabetizado ecológicamente a "comprender los principios de organización de los ecosistemas y utilizar dichos principios para crear comunidades humanas sostenibles" (Capra, 1996, p. 307) y con ello sus creaciones apoyadas en la biomimesis responden a un equilibrio natural. ${ }^{11}$

Del mismo modo, Capra hace notar que los ecosistemas funcionan con base en "Ciclos cerrados de la materia, movidos por la energía del sol" y esta es entonces una "economía cíclica" totalmente renovable y auto reproductiva, sin residuos, que utiliza el sol como fuente de energía inagotable en términos humanos. De allí se desprende la idea de Richmann (2000) que plantea la reconstrucción de los sistemas de producción imitando la producción natural de la biosfera, y señala como insostenibles los sistemas lineales de la industria capitalista desarrollada.

Diversos centros de investigación y grupos interdisciplinarios han desarrollado estrategias y métodos de aproximación biomimética para la resolución de problemas actuales, intentando ir más allá de la reproducción y/o simulación de organismos o partes de ellos, y acercándose a la reproducción de los ecosistemas. Entre ellos están Janine Benyus y Dayna Baumeister en el Biomimicry Institute, quienes utilizan lo que han denominado Espiral Biomimético. Esta "espiral de diseño" sirve de guía a los innovadores para usar la biomimética, biologizando los problemas de diseño, y evaluar al final si en realidad el producto imita a la naturaleza en todos los niveles (forma, proceso y ecosistema). 
Seis etapas constituyen la estrategia analógica del "espiral biomimético", en la que se desarrolla un planteamiento de diseño sobre una necesidad humana. Este planteamiento consiste en descomponer, disgregando el problema en sus partes esenciales, y así generar las especificaciones de diseño; identificar la función que se quiere lograr hasta encontrar lo que realmente el diseño debe implementar, y finalmente definir las especificaciones del problema que implica conocer el contexto y los actores que se relacionan con el problema.

Desde el diseño industrial hay un enorme interés en el seguimiento de modelos y estrategias como la Biomimesis dado su compromiso en el desarrollo material del entorno humano sin incrementar el deterioro ambiental y, por el contrario, contribuyendo con soluciones a problemas comunes que aporten a la conservación de la biosfera. Un camino de deterioro que desde la revolución industrial ha tomado más de 100 años no se podrá resolver a pesar de los avance de la ciencia en poco tiempo, pero que se debe convertir en un compromiso ineludible. Por ello se hacen necesarios estos primeros pasos.

\section{Proceso de diseño biomimético}

El proyecto "Simulador biomimético de la locomoción animal terrestre" del grupo de Desarrollo, Innovación e Investigación en Diseño, de la Universidad del Valle, tuvo como objetivo documentar el proceso de diseño, desarrollo y construcción de modelos funcionales de simulación de los mecanismos de locomoción animal terrestre como referencia del ejercicio biomimético y su reflexión como estrategia alternativa de diseño sustentable, orientado a la búsqueda de soluciones alternativas al transporte humano, con los siguientes propósitos: promover el uso de estrategias y métodos alternativos al abordar problemas de diseño, retomar el estudio de la naturaleza desde la perspectiva del diseño como referente "Natural" de la sustentabilidad, aplicar la estrategia de diseño "Espiral Biomimética” en procesos de simulación como base para las aplicaciones en diseño industrial y la construcción de modelos funcionales de simulación de los mecanismos de locomoción animal terrestre.

Asumiendo que las categorías funcionales de la locomoción animal terrestre está ligada a diversos aspectos fisiológicos o ecológicos como la dispersión de las especie, sea esta pasiva o activa, la migración (inmigración o emigración), el encontrar refugio, la búsqueda de alimentos y captura de presas, el huir de depredadores y enemigos, y el cortejo y apareamiento, entre otros, es necesario el estudio de la locomoción, incluyendo el estudio de los órganos locomotores o los sistemas más o menos pasivos de transporte y los mecanismos sensoriales que intervienen en la orientación, regulación y reconocimiento del camino adecuado. 
Estos estudios distinguen, la motilidad, uno de los rasgos característicos de la vida animal, a tener en cuenta en cada uno de los tipos de la actividad motriz; la locomotora cuando el animal se traslada a lo largo de un trayecto; la postural cuando las diferentes partes del cuerpo adoptan posiciones distintas, y la transvectorial, cuando no es el animal el que se traslada, sino que su actividad motriz conduce al desplazamiento del medio exterior. Para el caso de este proyecto no se tuvieron en cuenta los movimientos plasmáticos propios de ciertos protozoos rizópodos ni el movimiento vibrátil de los agelados o ciliados, y se desarrolló especialmente con el tipo de movimiento muscular, típico de los animales pluricelulares, que generan un sistema de músculos locomotores adecuados y tienen movimientos con ondas de contracción muscular y un sistema de palancas típico de patas articuladas en artrópodos y vertebrados.

Los mecanismos funcionales ligados a la modalidad de locomoción por sistema de palancas permiten reunir el movimiento marchador sobre patas articulares (marcha, carrera y salto) que es el propio y más común de todos los vertebrados que no son nadadores o voladores, y el de patas de los artrópodos, cuyo nombre precisamente significa "patas articuladas", y el sistema de palancas de tipo hidrostático.

Desde el punto de vista ecológico los animales que no son fijos o muy sedentarios - y éstos en general presentan larvas o estadios juveniles móviles - utilizan sus mecanismos locomotores para la colonización de aquellos ambientes que les son más favorables. Además la rapidez o estilo peculiar de la marcha, lo que podemos llamar andadura, que depende de los múltiples factores del medio en que el animal se mueve, de su aparato locomotor, muscular, nervioso y sensorial, y de los estímulos que condicionan y guían la locomoción, son tan específicos que permiten diferenciar de modo claro unos animales de otros.

La andadura no cambia con facilidad, pero bajo una variación ecológica puede alterarse. La andadura específica, por ejemplo el paso, trote y galope en un caballo, la marcha rastreante, sobre sus brazos o la huida por propulsión a chorro en el pulpo, son producto de estímulos ecológicos específicos, la simple traslación o el miedo, en estos casos.

Para el desarrollo del estudio se determinaron criterios y categorías como éxito biológico, prevalencia de artrópodos en la tierra y ventaja mecánica del exoesqueleto, y, para cada una, se identificaron los especímenes que cumplieran también con ser totalmente singulares o aquellos que son muy similares. Así se generaron grandes grupos como Saltadores, Caminadores, Peristálticos, Mediante ondas y mediante Líquidos no Newtonianos. 
La selección final de especímenes con base a los criterios y grupos fueron:

\begin{tabular}{|c|c|c|c|c|}
\hline Grupo & Familia & Especie & \# de Patas & Característica \\
\hline 1 & Arácnidos & $\begin{array}{l}\text { Tarántula } \\
\text { Escorpión }\end{array}$ & $\begin{array}{l}8 \\
8\end{array}$ & $\begin{array}{l}\text { Cadencia y gestión del } \\
\text { movimiento articular }\end{array}$ \\
\hline \multirow{2}{*}{2} & \multirow{2}{*}{ Insectos } & Cucarrón & 6 & \multirow{2}{*}{$\begin{array}{l}\text { Tipo de articulación común } \\
\text { de rotación }\end{array}$} \\
\hline & & Saltamontes & 6 & \\
\hline 3 & Miriápodos & & N. & Pisada sobre pisada \\
\hline 4 & Crustáceos & Cangrejo & 8 & $\begin{array}{l}\text { Singularidad en la dirección } \\
\text { del movimiento }\end{array}$ \\
\hline 5 & Gasterópodos & & 0 & $\begin{array}{l}\text { Singularidad en la técnica } \\
\text { del movimiento }\end{array}$ \\
\hline 6 & Oligoquetos & & 0 & $\begin{array}{l}\text { Singularidad en el } \\
\text { mecanismo del movimiento }\end{array}$ \\
\hline \multirow{2}{*}{7} & Anfibios & Sapo & 4 & \multirow{2}{*}{$\begin{array}{l}\text { Las patas al mismo nivel de } \\
\text { la columna }\end{array}$} \\
\hline & Reptiles & Lagartija & 4 & \\
\hline 8 & Aves & Avestruz & 2 & Único bípedo \\
\hline \multirow{4}{*}{9} & \multirow{4}{*}{ Mamíferos } & Danta & 4 & \multirow{3}{*}{$\begin{array}{l}\text { Todos los cuadrúpedos. } \\
\text { Todas las articulaciones bajo } \\
\text { la columna }\end{array}$} \\
\hline & & Puma & 4 & \\
\hline & & Caballo & 4 & \\
\hline & & Canguro & 2 & Único mamífero saltador \\
\hline
\end{tabular}

Tabla 1.Análisis de grupos de especies animales según algunas características que definen su desplazamiento.

Para cada espécimen se sintetizo en fichas descriptivas los rasgos generales y aquellos relacionados con la interacción en el entorno y su mecanismo de locomoción. También se presenta el análisis de sus movimientos articulares determinando las articulaciones tenidas en cuenta, señalando el orden en que se han considerado para su medición desde la vista frontal y superior o lateral según sea el caso, y el estudio, a partir de los registros de tiempo 
de cada uno de sus miembros, de las diferentes fases de la marcha para los distintos especímenes. Se tuvieron en cuenta, el inicio del levante, el levante, inicio de apoyo y el apoyo (en quienes lo incluyen).

Adicionalmente se presentan las gráficas de diagrama de marcha en donde se evidencian los ritmos, tiempos y alternaciones en el apoyo de sus miembros durante la marcha y finalmente el diagrama de Muybridge que nos muestra gráficamente las fases de la locomoción.

\begin{tabular}{|l|l|}
\hline Reino: Animal \\
Filo: Chordata \\
Clase: Amphibia \\
Orden: Anura \\
Familia: Bufonidae \\
Género: Bufo \\
Especie: Bufo bufo
\end{tabular}

\section{Descripción general:}

Los sapos son vertebrados pertenecientes a la clase de los anfibios, que se caracterizan por su larga lengua y sus saltos; su cuerpo es corto y rígido, su cabeza grande y ancha; sus extremidades delanteras son más cortas que las traseras y se caracterizan por contar con sección adicional compuesta por los huesos calcáneo y astrágalo. La musculatura de los sapos se ha desarrollado principalmente para la función de locomoción. En estos animales las extremidades posteriores están adaptadas para el salto por lo que cuentan con músculos especializados, que proporcionan mayor volumen muscular.

Los sapos se mueven mediante diferentes formas. La mayoría de los sapos son terrestres y caminan, corren y saltan sobre el suelo. Sin embargo los sapos no utilizan el salto para trasladarse, ya que muy pocos no son capaces de saltar grandes distancias, y lo usan principalmente para escapar de sus depredadores, es decir como medida defensiva. Por otra parte no todos los sapos saltan, algunos de ellos han especializados sus patas posteriores para nadar o escalar, también han desarrollado en su patas una alta capacidad excavadora, para poder refugiarse en agujeros profundos o debajo de troncos o rocas.

En las patas posteriores cuentan con 5 dedos y en las anteriores con solo 4 y varían según el estilo de vida de la especie; algunos de hechos han desarrollados membranas entre sus dedos que les permite nadar mejor, otros trepadores, poseen cojines en forma de discos adhesivos para sujetarse a diferentes superficies y aquellos que principalmente escavan, poseen una callosidades llamadas tubérculos para facilitarles la tarea.

La marcha que realiza el sapo se da de manera cíclica, permitiendo entonces articular rítmicamente su movimiento. La velocidad depende del terreno en que se esté desplazando, este desplazamiento posee una velocidad constante y para las superficies irregulares su velocidad es más controlada y tiende a levantar mucho más sus patas al caminar, para acelerar el desplazamiento lo realiza a través del salto. Cuando realiza pausas en su movimiento sus patas quedan en la posición en la cual se encuentra durante su marcha, al ubicarse en posición de reposo recoge su extremidades hacia su cuerpo para descansar sus patas.

Tabla 2. Ficha Tipo: Descripción de características relevantes para el análisis de especies según sus características de locomoción. 
Se procede luego a realizar un análisis de los movimientos de los especímenes animales seleccionados de modo que sea posible describir su movimiento articular y su secuencia de movimientos en cuanto a apoyos y tiempos. Para esto se realiza un estudio mediante diagramas obtenidos del análisis de imagen fija e imagen en movimiento. Se desarrolla: 1. Análisis de movimiento articular, 2. análisis gráfico de movimiento articular 3. análisis de marcha, 4. diagrama de marcha y 5. diagrama de Muybridge.

\section{Análisis de movimiento articular}
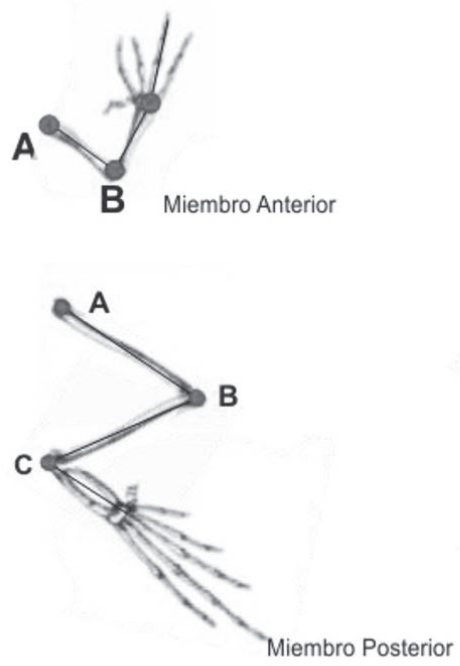

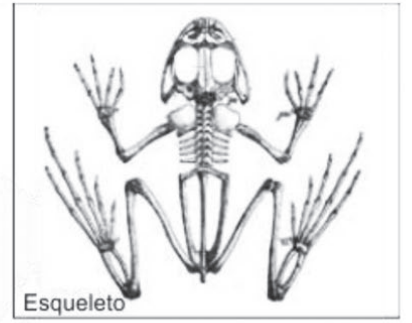

B

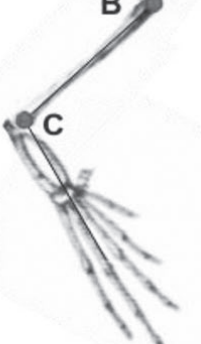

Miembró Posterior Maxima extensión el el salto

\begin{tabular}{|c|c|c|c|c|c|}
\hline \multirow{2}{*}{$\begin{array}{c}\text { Articulación/ } \\
\text { Junta }\end{array}$} & \multirow{2}{*}{ Tipo de Junta } & \multicolumn{4}{|c|}{$\begin{array}{c}\text { Selección del Miembro } \\
\text { (Ángulo barrido de movimiento relativo) }\end{array}$} \\
\cline { 2 - 6 } & & Anterior & $\begin{array}{c}\text { Medio } \\
\text { Anterior }\end{array}$ & $\begin{array}{c}\text { Medio } \\
\text { Posterior }\end{array}$ & Posterior \\
\hline A & 5 & $140^{\circ}$ & - & - & $90^{\circ}$ \\
\hline B & 11 & $100^{\circ}$ & - & - & $112^{\circ}$ \\
\hline C & 11 & - & - & - & $104^{\circ}$ \\
\hline
\end{tabular}

Esquema de juntas: 1. Rotación 2. Prismática 3. Helicoidal 4. Cilíndrica 5. Esférica 6. En plano 7. Pasador completa para rotación (con cierre de forma) 8. Corredera completa para traslación (con cierre de forma) 9. Eslabón apoyado contra un plano (con cierre de fuerza) 10. Pasador en ranura (con cierre de forma) 11. Pasador de Primer orden con un GDL (dos eslabones conectados) 12. Pasador de segundo orden con dos GDL (dos eslabones conectados) 13. Rodamiento puro,

Tabla 3. Análisis de movimientos articulares de especies animales. 


\section{Análisis gráfico de movimientos articulares.}

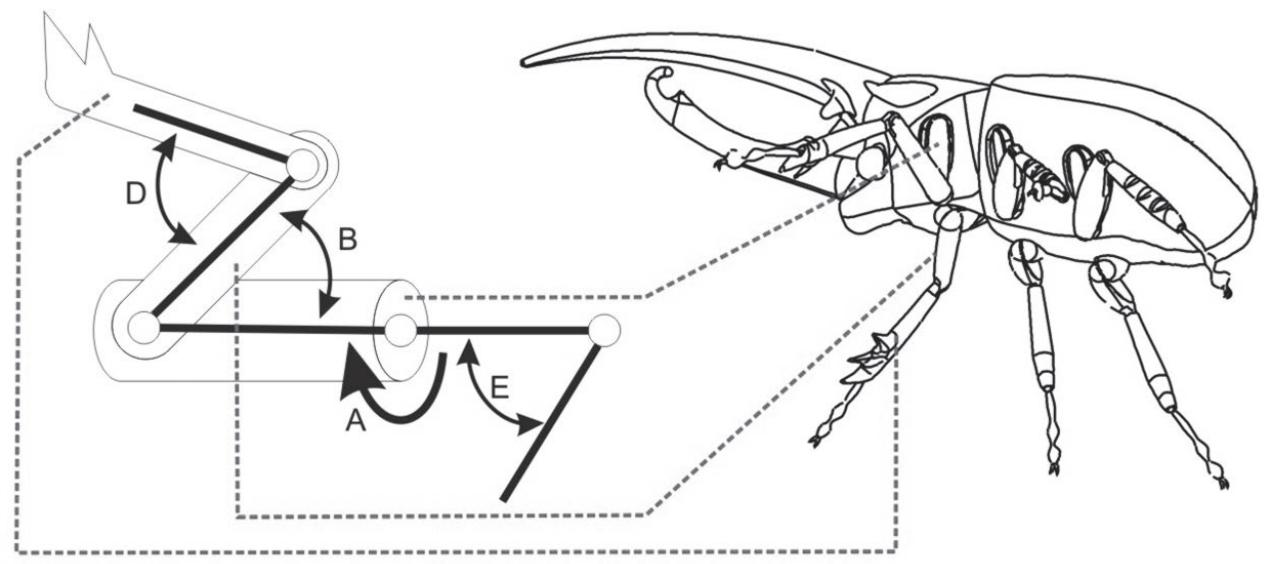

Figura 2. Análisis gráfico de movimientos articulares. (Uribe, 2013). Se observa la definición de los movimientos relativos entre segmentos de la pata y la clasificación del tipo de junta.

\section{Análisis de marcha}

\begin{tabular}{|c|c|c|c|c|}
\hline & Inicio Apoyo & Apoyo Completo & Inicio Levante & Levante Aire \\
\hline Miembro Superior Derecho & $\begin{array}{l}1: 04 \\
2: 06 \\
3: 08 \\
4: 08 \\
5: 10 \\
6: 12\end{array}$ & $\begin{array}{l}1: 06 \\
2: 08 \\
3: 10 \\
4: 10 \\
5: 12 \\
6: 14\end{array}$ & $\begin{array}{l}1: 16 \\
2: 18 \\
3: 20 \\
4: 20 \\
5: 22 \\
6: 24\end{array}$ & $\begin{array}{l}1: 22 \\
2: 24 \\
4: 00 \\
5: 00 \\
6: 02 \\
7: 04\end{array}$ \\
\hline Miembro Superior Izquierdo & $\begin{array}{l}0: 15 \\
1: 17 \\
2: 19 \\
3: 21 \\
4: 23 \\
5: 23\end{array}$ & $\begin{array}{l}0: 17 \\
1: 19 \\
2: 21 \\
3: 23 \\
5: 00 \\
6: 00\end{array}$ & $\begin{array}{l}1: 03 \\
2: 05 \\
3: 07 \\
3: 09 \\
5: 10 \\
6: 10\end{array}$ & $\begin{array}{l}1: 09 \\
2: 10 \\
3: 13 \\
3: 15 \\
5: 16 \\
6: 16\end{array}$ \\
\hline Miembro inferior Derecho & $\begin{array}{l}0: 16 \\
1: 18 \\
2: 21 \\
3: 21 \\
4: 23 \\
5: 23\end{array}$ & $\begin{array}{l}0: 18 \\
1: 20 \\
2: 23 \\
3: 23 \\
5: 00 \\
6: 00\end{array}$ & $\begin{array}{l}1: 06 \\
2: 08 \\
3: 10 \\
4: 10 \\
5: 13 \\
6: 13\end{array}$ & $\begin{array}{l}1: 12 \\
2: 15 \\
3: 17 \\
4: 17 \\
5: 19 \\
6: 19\end{array}$ \\
\hline Miembro inferior Izquierdo & $\begin{array}{l}0: 06 \\
1: 07 \\
2: 09 \\
3: 10 \\
4: 10 \\
5: 11\end{array}$ & $\begin{array}{l}0: 08 \\
1: 09 \\
2: 11 \\
3: 12 \\
4: 12 \\
5: 13\end{array}$ & $\begin{array}{l}0: 21 \\
1: 23 \\
2: 24 \\
4: 00 \\
5: 00 \\
6: 01\end{array}$ & $\begin{array}{l}1: 01 \\
2: 02 \\
3: 04 \\
4: 05 \\
5: 06 \\
6: 06\end{array}$ \\
\hline
\end{tabular}

Tabla 4.Análisis de tiempos en el desplazamiento de diferentes especies animales. 


\section{Diagrama de marcha}

MSI

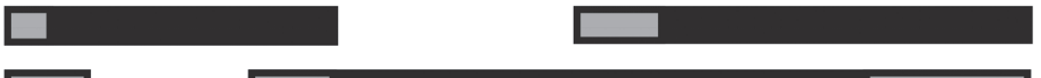

MII

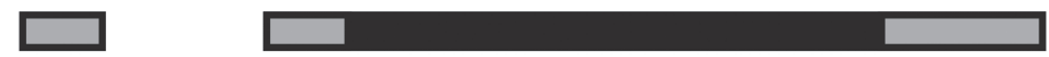

MSD

MID

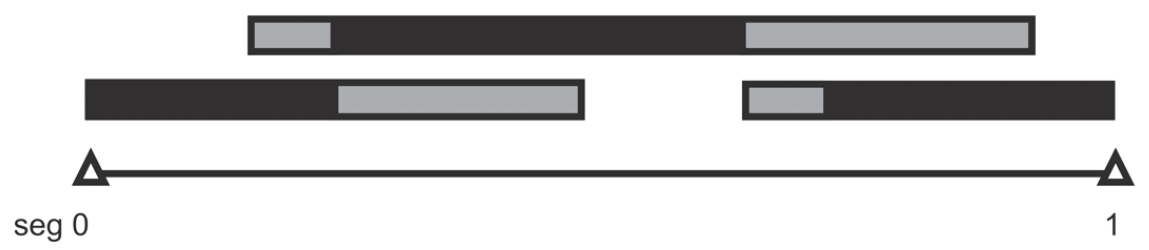
MSI: miembro superior izquierdo
MII: miembro inferior izquierdo
MSD: miembro superior derecho
MID: miembro inferior derecho

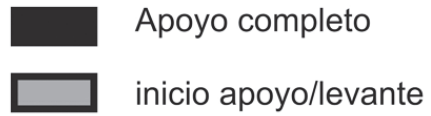

Figura 3. Análisis simplificado de tiempos y apoyos de extremidades de especies animales en un ciclo de locomoción.

Diagrama de Muybridge
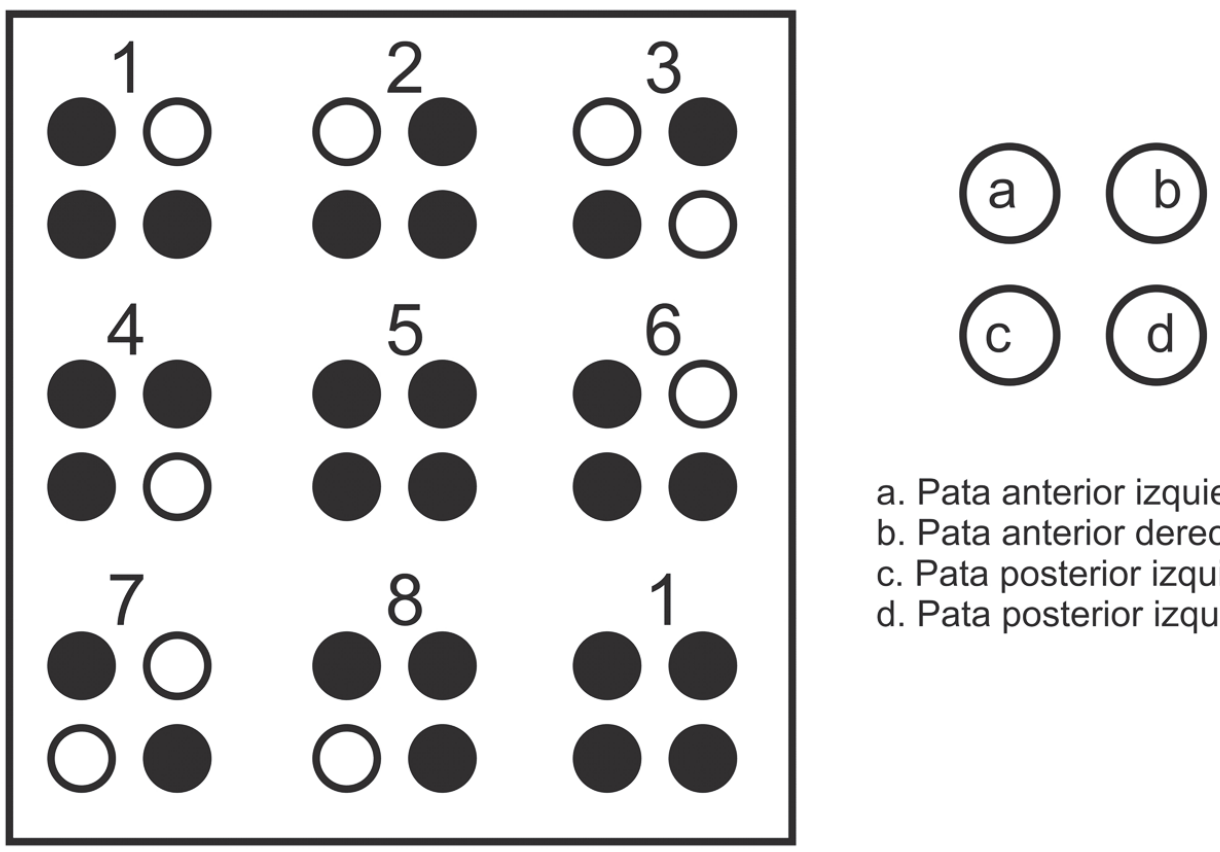

a. Pata anterior izquierda

b. Pata anterior derecha

c. Pata posterior izquierda

d. Pata posterior izquierda

Figura 4. Análisis mediante el diagrama de Muybridge. Secuencia de apoyos de extremidades en 8 momentos de la marcha. 
Aun cuando funcionalmente los mecanismos de locomoción animal terrestre se corresponden más con las formas de simulación por eventos discretos dado que el comportamiento varía tanto en instantes del tiempo como en la conformación por la acomodación a la superficie, lo clave es la simulación de las variaciones y no del principio funcional fundamental, si tenemos en cuenta que los momentos en los que se producen los cambios son los que se identifican como los eventos del sistema o simulación. De tal modo que otorga mayor control y por tanto aumenta las posibilidades de éxito la simulación por autómatas celulares que se aplica a casos complejos, como este, en el que se divide el comportamiento del sistema en subsistemas más pequeños denominados células, intentando hacerlo para aquellas que representan el principio funcional fundamental. Así y de ser necesario el resultado de la simulación estará dado por la interacción de las diversas células.

El mecanismo simulador de barridos articulares, desarrollado a partir de la metodología de autómatas celulares, permitió de manera aproximada establecer tanto los ángulos recorridos como las longitudes de avance, retroceso y altura alcanzada de cada punto dentro de la articulación y para cada combinación de radios y longitudes de los cuatro segmentos articulares construidos. El diseño de la pierna articulada forma parte del proceso de esta investigación y busca resolver el mecanismo de la manera más simple posible, sin la necesidad de un actuador en cada articulación para lograr movimientos coordinados (Uribe, 2013)

\section{Mecanismo simulador}
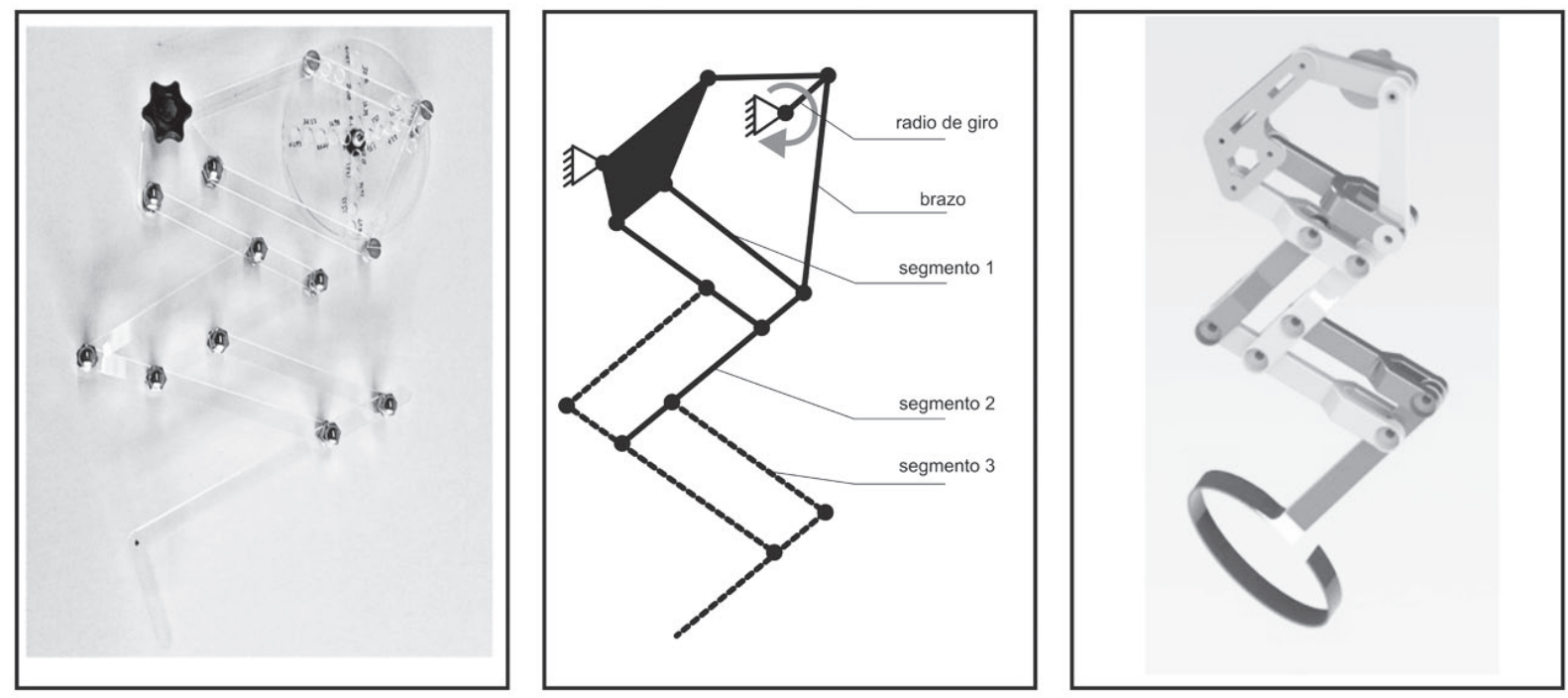

Figura 5. Diseño de mecanismo simulador biomimético de para articulada.

Se trata de un mecanismo de barras paralelas en secuencia. Un mecanismo simple y mecánico que no requiere actuadores en las articulaciones para lograr movimientos coordinados de los segmentos. 
El mecanismo de simulación consta de un plato de giro que determina la amplitud de los movimientos generales, dos brazos que transmiten el movimiento a los segmentos principales y determinan el avance, retroceso y altura de los movimientos y cuatro segmentos que llevan al límite máximo y mínimo el desplazamiento del simulador.

Se llevó a cabo una experimentación práctica con el mecanismo diseñado realizando variaciones en las dimensiones de radio de giro del plato y extensión de los brazos; los resultados de cada una de estas combinaciones se registraron fotográficamente y gráficamente con los datos correspondientes y se realizó un registro de las mismas.

\section{Registro tipo.}
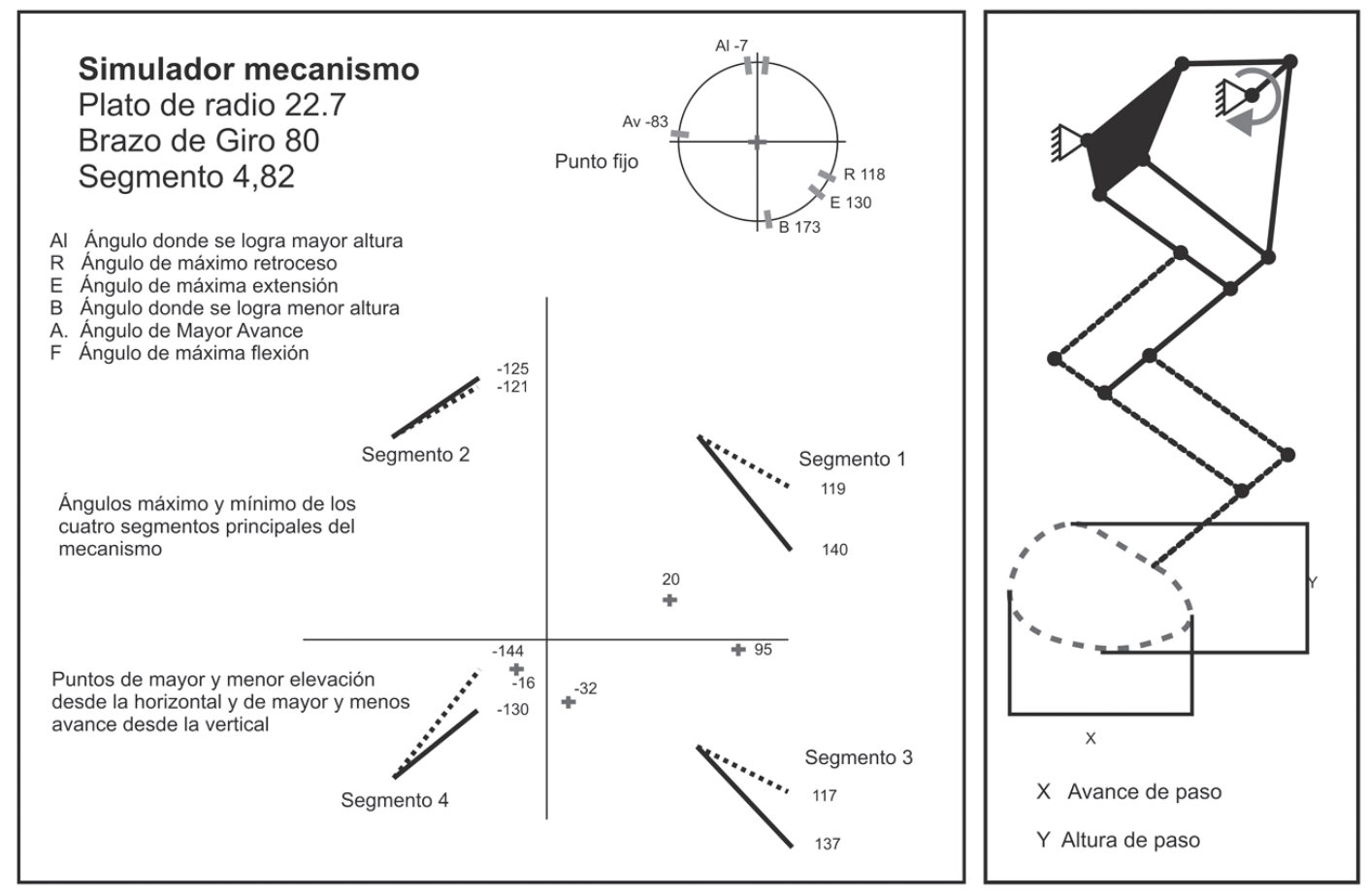

Figura 6. Registro de movimientos del mecanismo simulador biomimético de pata articulada.

El mecanismo de barras paralelas permite mediante la variación de longitudes de sus segmentos, lograr diferentes formas de movimiento, diversas alturas del paso y diversas longitudes o distancia máxima de paso en cada ciclo.

Con tres longitudes fijas para cada uno de los cuatro segmentos articulares y catorce radios de giro se obtuvieron 492 gráficas con 16 datos específicos de posición que permitieron medir cada distancia para cada posición de las articulaciones. 


\section{Análisis de resultados comparados}

La gran cantidad de datos y resultados del ejercicio de medición del mecanismo de simulación, nos permite un amplio marco de comparación de variables, desde la afectación de los ángulos máximo y mínimo de los cuatro segmentos, hasta las variaciones de las dimensiones en las longitudes de referencia (avance máximo y mínimo o retroceso en relación al punto cero y altura máxima y mínima desde el punto cero); así mismo las afectaciones de ellas en la variación de las longitudes tanto de los brazos de giro como la de los segmentos del mecanismo. De tal forma que presentamos aquí mediante un análisis gráfico simplificado solamente algunas de las conclusiones relevantes para la construcción del simulador.

Una representación mediante ciclogramas permite visualizar el movimiento de la parte distal de la pata en el mecanismo, Uribe (2011) analiza los métodos de representación de la locomoción animal y presenta este tipo de diagramas donde se interpreta el eje horizontal como el tiempo de la marcha y se define el recorrido articular mediante líneas. El siguiente diagrama usa este método de representación para el análisis de resultados.

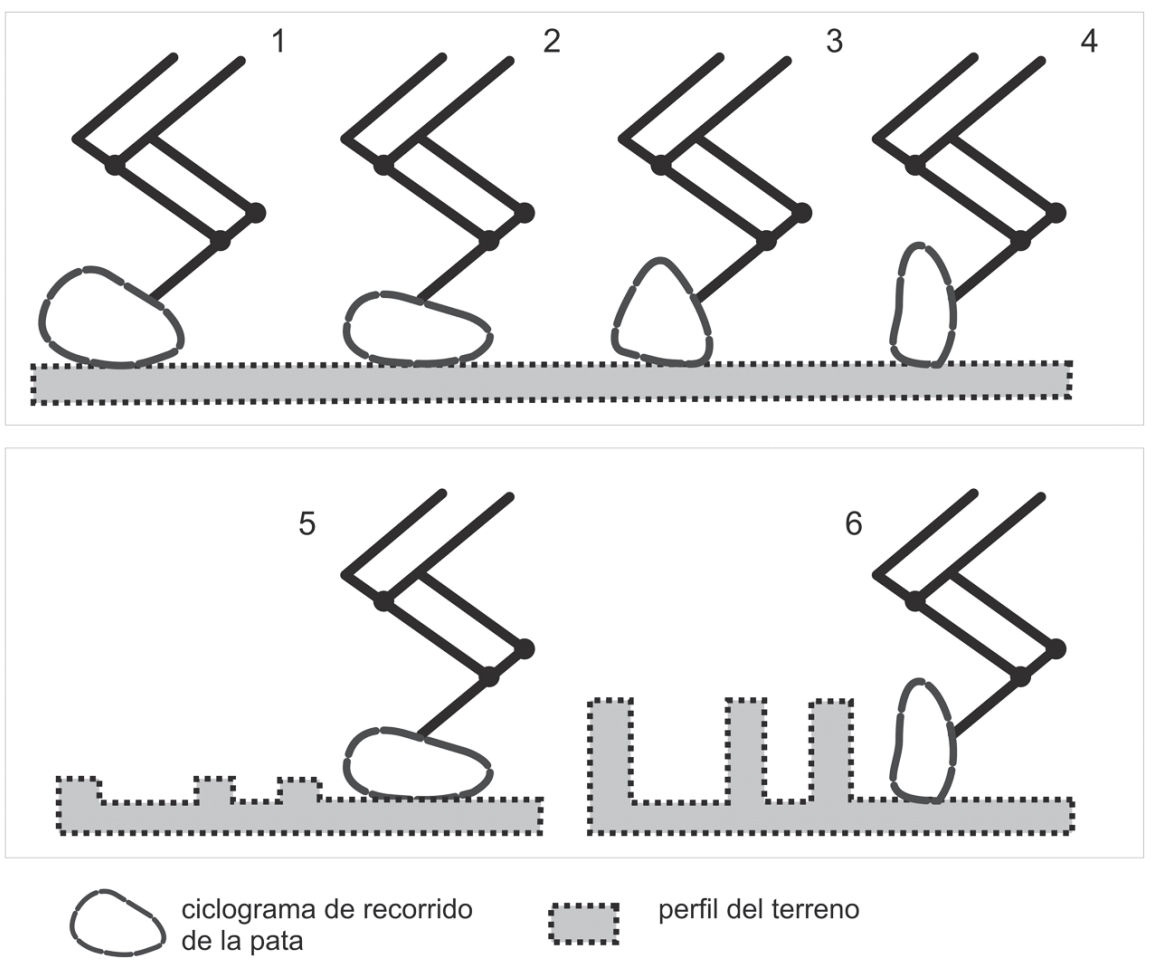

Figura 7. Análisis comparativo de diversas formas de paso logradas mediante la variación de medidas en el mecanismo de simulación de pata articulada diseñada. (se observa variación de los ciclogramas 1, 2, 3 y 4). El mecanismo permitirá superar obstáculos de diversas medidas, ya que como resultado de la variación del mismo se logran formas de movimiento más altas y cortas(5 - para obstáculos altos) o más largas y bajas (6 - para obstáculos mas bajos). 


\section{Conclusiones}

1. A pesar del avance en cuanto a la disminución de impacto ambiental negativo por el desarrollo de motores de combustión interna más eficientes y fuentes alternas de energía, el paradigma de movilidad mediante rueda está condicionado a la intervención del terreno que necesita ser preparado con este fin causando una importante huella ecológica.

2. Los organismos naturales logran altísimos niveles de eficiencia en relación al consumo energético y una enorme capacidad de adaptación a terreno sobre el que se desplazan, esto a pesar de haberse concentrado el proyecto en la mera simulación del movimiento articular.

3. Esta etapa de la investigación demostró la posibilidad de generar desplazamientos a partir de elementos mecánicos, relacionados con los segmentos articulares de los animales estudiados.

4. En el mecanismo diseñado es posible ajustar el "paso" en términos de avance y altura de cada pata articulada.

5. Se hace evidente que es posible el desarrollo de un mecanismo simple que permiten seguir estudiando alternativas de desplazamiento para condiciones especiales o requerimientos específicos.

\section{Notas}

${ }^{1}$ Comparativa de la velocidad de los animales. http:/ / es.wikipedia.org/wiki/Velocidad_de_los_ animales 16-03-2015

${ }^{2}$ http: / / datos.bancomundial.org/indicador/IS.RRS.TOTL.KM

${ }^{3}$ http: / / asimo.honda.com

${ }^{4}$ http: / / global.kawada.jp/mechatronics/hrp4.html

${ }^{5}$ https://www.aldebaran.com/en/humanoid-robot/nao-robot

${ }^{6}$ http: / / www.tmsuk.co.jp

${ }^{7}$ http://www.bostondynamics.com/robot_Atlas.html

${ }^{8}$ http: / / www.bostondynamics.com/robot_ls3.html

${ }^{9}$ http: / / www.robugtix.com/t8/

${ }^{10}$ https: / / www.grasp.upenn.edu

${ }^{11}$ Los cinco principios enunciados por Capra para estar eco alfabetizado son: 1. Independencia 2. Naturaleza cíclica de los procesos biológicos 3. Tendencia a asociarse, establecer vínculos y cooperar como características esenciales de la vida 4. Flexibilidad y 5. Diversidad. 


\section{Referencia}

Capra, F. (1996). La trama de la vida, una nueva perspectiva de los sistemas vivos. New York, New York, USA: Anagrama.

Carthy, J. D. (1971). La conducta de los animales. Barcelona, Cataluña, España: Salvat.

Cromer, A. H. (1996). Física para las ciencias de la vida. Barcelona, Cataluña, España: Reverté.

Georgescu-Roegen, N. (1971). La ley de la antropía y el proceso económico. Madrid, España: Antonio Machado.

Maliandi, R., \&Thüer, O. (2008). Teoría y praxis de los principios bioéticos. Remedios de la escalada, Buenos Aires, Argentina: Universidad nacional de Lanus.

Millares Marrero, R., \& Puig Cunillera, M. (1998). Biomecánica clínica del aparato locomotor. Barcelona , Cataluña , España: Masson.

Nordin, M., \& Frankel, V. (2001). Biomecánica básica del sistema musculo esquelético. Madrid, España: Mc Graw Hill.

Riechmann, J. (2000). Un mundo vulnerable. Madrid, España: Los libros de la catarata.

Uribe, Miguel. (2011). Representación de la locomoción animal terrestre como estrategia de análisis de movimiento para el desarrollo de simuladores biomiméticos. En: Revista Nexus, No. 10.

Uribe, Miguel. (2013). Diseño de pierna articulada para el planteamiento de medios alternativos de transportes. En: Revista Nexus, No. 12.

Recibido: septiembre 30 / Aprobado: noviembre 20 de 201 